\title{
Problems and Improvements in English Translation of Export Food Packaging Texts
}

\author{
Ma Qinhua \\ School of Foreign Studies, Xi'an University, Shaanxi, China, 710065
}

Keywords: Export Food Packaging; English Translation; Food Enterprises; Consumers.

Abstract: Because the English translation of export food packaging cannot be strictly managed at present, a lot of problems have arisen in the actual operation, which is not conducive to improving the market competitiveness of export food. The main reasons are the large use of translation tools, the lack of attention to the study of grammar, the lack of attention to the analysis of the cultural customs of importing countries, the lack of professionalism of translators, the lack of strict scrutiny, and the lack of knowledge of the taboos of importing countries. In order to solve this problem, it is necessary to analyze the characteristics of English itself and the cultural characteristics of the importing countries in order to find solutions to the problem.

\section{Introduction}

Food packaging does not only have a certain protective effect on food, but also is used for transportation services. Consumers can understand all kinds of information of food on the text of self-packaging. The packaging of export food must be printed in English. First, it can provide convenience for foreign consumers and facilitate their choice. Secondly, consumers can understand the internationalization degree of products from packaging, satisfy the consumer psychology of the public to achieve consumer satisfaction. Due to some defects in the current management of export food, many problems inevitably arise in the actual operation, which seriously restrict the international competitiveness of export food, and even lead to the phenomenon of returns from purchasers, which further triggers international contradictions and causes great economic losses to Chinese food enterprises. Based on in-depth study of these problems, it is of great practical significance to formulate corresponding measures to solve the problem of non-standard translation of export food packaging English ${ }^{[1]}$.

\section{A Basic Summary of English Translation of Food Packaging Texts}

Food packaging text mainly involves food name, nature, use method, product technology, matters needing attention, etc. Only by ensuring that the translation process is accurate, simple and clear, can it be recognized by consumers. From these words, consumers can also find the propaganda strength of enterprises and the understanding of food culture. In terms of self-confidence and information dissemination, food packaging can also serve as a reminder to educate consumers. In a word, food packaging text contains all kinds of information related to food 
and food production enterprises. It is a sign of introducing food, so it has high requirements for English translation. Food packaging mainly includes text, letters, numbers, lines, graphics, pictures and other contents. Foods entering the Chinese market should be packaged with food names, nutrients, product standards, production licenses, storage and use methods, enterprise information, etc. All information must be reflected in Chinese. In order to further achieve the effect of promoting food, some enterprises print English in the corresponding position in Chinese, which is also a good way to present both Chinese and English ${ }^{[2]}$. Firstly, improve the scope of information dissemination and achieve international development. Second, improve the corporate image and stimulate consumers to buy. Among the foods exported from other countries, all the words on the package are required to be displayed in English except for the food trademarks, and the food trademarks are still displayed in Chinese. If an enterprise plans to build a brand in an importing country, it must register an English trademark with the same meaning as Chinese in the corresponding country. The detail translation process of export food packaging texts shown as below figure 1 .

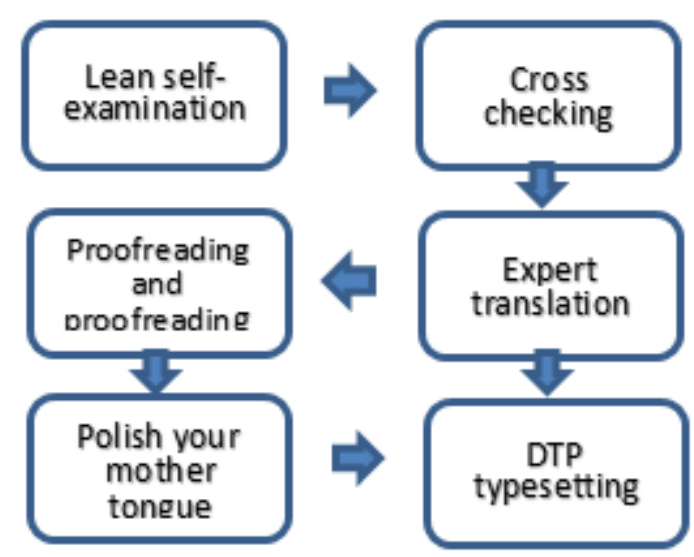

Figure 1 Translation process of export food packaging texts

\section{Adverse Factors in English Translation of Export Food Packaging}

\subsection{Translation is too mechanical}

When translating food packaging, the most errors are that the translation method is mainly mechanical, without considering the influence of other factors. To translate Chinese into English, it is necessary to ensure that English and Chinese can achieve the same effect in different language application environments ${ }^{[3]}$.

However, there are many mistakes in the application of mechanical literal translation, which will lead to many misunderstandings and even jokes among consumers in English-speaking countries. For example, "onion flower pancake" produced by a food company is translated into English and translated into "onion flower pancake". That is to say, the three words "onion", "flower" and "pancake" are translated directly, without considering the actual language environment, which makes people laugh and laugh. In this process of translation, every English word corresponds to the corresponding Chinese characters, without any mistakes. However, due to the mechanical literal translation method cannot pay attention to the different words of Chinese characters, the meaning changes when they are grouped together. "Pancake" means pancake, which is correct, but if "onion flower" is translated as "onion flower", there is a problem. In Chinese vocabulary, onion flower is a special noun, not "onion flower", which is quite different from "onion flower". Therefore, this kind of translation cannot express the real meaning of words in different languages. Different Chinese nouns will show different meanings when combined, so it is too hasty to use such a translation method. 


\subsection{Grammatical errors arise}

English translation on food packaging should be consistent with English grammar in order to help people understand the meaning correctly. There are some differences between Chinese grammar and English grammar. Some grammatical errors occur in the translation of English on food packaging. When consumers see them, they fall into a cloud, which is not conducive to the sale of goods. If some food is required to be kept in a cool place, some food companies use the following English language when translating English into food packaging: "Please put it in a com placent seal save". According to English grammar, "to" is not a preposition, but a combination of the following verbs to form an infinitive, followed by an infinitive. "Seal in" is a gerund, which obviously contradicts English grammar. Meanwhile, mechanical literal translation still exists in seal save, which is in contradiction with English usage. I think it should be translated as "Please put it in a cool place sealed to aver". When such grammatical errors occur, most consumers can guess the true meaning of their expressions after seeing English, but they still feel very uncomfortable, and even some consumers will choose to give up ${ }^{[4]}$.

\subsection{Understanding the Folklore Culture of Importing Countries}

Language embodies the local cultural characteristics. Different kinds of languages can also contain different cultural contents. Some food packages fail to recognize the importance of their cultural content in the process of English translation, which leads consumers in English-speaking countries to fail to understand their meanings and even arouse public disgust. For example, the famous Chinese confectionery brand "Big White Rabbit", many people think that its packaging can be directly translated as "the big white rabbit". But in practical application, there is a word "white rabbit" in English which means like "big rabbit" in China. When "big" is added to the translation process, consumers in English-speaking countries interpret it as "big white rabbit", which causes the instant collapse of the brand image of food and the fundamental feeling of consumers. Cannot stand the "big white rabbit" lovely. Moreover, many Chinese food brands contain the word "dragon", while some export enterprises translate it directly into English "dragon" on food packaging. But although Chinese and English "dragon" have similar meanings, Chinese people regard dragon as an auspicious animal, but British people regard dragon as a vicious beast, which has a certain derogatory meaning. If consumers in English countries see the brand of "dragon", will they choose to buy it? Many enterprises in China's food industry are named Fangfang. In the packaging of export food, some enterprises directly translate Fangfang into Fangfang. But in English, "Fang" is a kind of poisonous teeth of animals. If consumers in English environment see the food brand of poisonous teeth, they will like and choose to buy it. The result is self-evident ${ }^{[5]}$.

\section{Strategies for English Translation of Export Food Packaging}

\subsection{Food manufacturers should attach great importance to English translation of food packaging}

The main reason why there are many problems in English translation of food packaging is that it has not attracted the attention of food manufacturers. Such as blindly applying translation software, not hiring high-level translators in time, not knowing the cultural customs of the exporting countries, and not strictly checking English translation, these are the main factors causing frequent English translation problems in food production enterprises. Therefore, food manufacturers should attach great importance to English translation of food packaging in order to solve this problem fundamentally. The author believes that food manufacturers can use the following methods to solve 
this problem: Firstly, training and education for the existing English translators of food packaging, requiring all translators and decision makers to participate, can arouse these staff's great attention to the English translation of food packaging. In order to prevent similar mistakes in actual operation, we should adjust our working ideas in time and make clear the direction of our efforts. Secondly, a sound auditing system should be established. After the first draft of food packaging English is completed, it must be strictly audited by professionals. Once problems are found, they should be corrected in time to prevent the problem of English translation of food packaging into other countries.

\subsection{Improving the Professional Skills of Translators}

Problems in English translation of food packaging are also related to the translator's personal competence. If the translator's professional skills are outstanding, he will translate "green onion pancake" into "onion flower pan" instead of "onion flower pan"

Cake ". Therefore, it is very important to improve the professional skills of translators. The author believes that the following measures can be taken: First, for the current English translators in food enterprises, training methods should be used to improve their professional knowledge level, requiring them to strengthen their learning, master the theoretical knowledge of English translation and improve their English. Translation skills. Secondly, if food enterprise translators are not sure whether the English translation is accurate or not, they may seek the help of professional translation department. Enterprise translators can strengthen communication with the staff of professional translation department and improve the professional quality of enterprise translators.

\subsection{Extensive solicitation of opinions to solve problems}

When confronted with uncertain food translation in the process of food English translation, we can solicit your opinions. If the results of food packaging English translation cannot be determined because of certain cultural differences, the relevant personnel are required to enter relevant countries to carry out on-the-spot investigations, and at the same time, a large number of investigations and studies are carried out in conjunction with the opinions of professional food English translators and market planners, so as to ultimately determine the English mode of food packaging. For example, the famous candy "Big White Rabbit", that is, after widely soliciting opinions from many participants, "white rabbit" is more appropriate, and quickly gained the favour of consumers after entering the target market.

\section{Conclusion}

In short, in recent years, China has exported many foods to other countries, and the English translation of export food packaging has attracted more and more attention. However, food manufacturers fail to recognize the importance of English translation of food packaging, resulting in numerous and complex problems, which seriously affect the international competitiveness of the enterprises concerned. Therefore, the problems in English translation of food packaging are explored in depth to conform to the characteristics of English itself and the cultural customs of the importing countries. Taking effective measures to solve these problems in time can achieve better results.

\section{References}

[1] Xu Keyao. Problems and Improvements in English Translation of Export Food Packaging Texts [J]. Overseas English, 2018 (14): 115-116. 
[2] Gong Yihao. On the Problems and Strategies of English Translation of Export Food Packaging Texts [J]. Taxation, 2017 (24): 186.

[3] Chen Yeyu. An analysis of English translation strategies of Quanzhou's export food instructions [J]. Examination and evaluation, 2016 (02): 137-138.

[4] Liu Xi. On Chinese-English Translation of Commodity Packaging: Guided by Equivalence Theory [J]. Overseas English, 2012 (07): 129-130.

[5] Dong Xinxinxin. Study on trade measures and Countermeasures of food labeling technology [D]. Chinese Academy of Agricultural Sciences, 2009. 